\title{
RETRACTED: Do anthocyanins in purple tomatoes reduce the risk of cardiovascular disease?
}

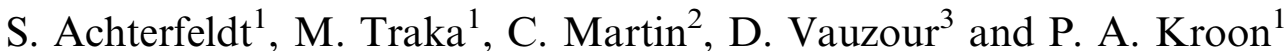 \\ ${ }^{1}$ Food and Health Program, Institute of Food Research, ${ }^{2}$ Metabolic Biology Department, John Innes Centre - Norwich \\ Research Park, Norwich NR4 7UH, UK and ${ }^{3}$ Medical School, University of East Anglia, Norwich Research Park, \\ Norwich NR4 7TJ
}

Anthocyanins $(\mathrm{ACN})$ are responsible for the red to dark purple colours in various fruits and vegetables. Epidemiological studies have shown an inverse relationship between consumption of ACN and risk of disease, particularly cardiovascular diseases (CVD). Supplementation of animal diets with ACN-rich foods and extracts has been shown to reduce atherosclerosis, improve vascular function and alter gene expression. However, these studies cannot prove that ACNs cause the observed effects because these foods and extracts contain many other potentially bioactive components apart from ACNs. The major challenge that has prevented researchers from directly linking ACNs in foods with beneficial effects in humans is the lack of suitable control foods (i.e. similar foods that lack anthocyanins). Such foods would also facilitate studies of the underlying mechanisms by which anthocyanins cause the observed effects.

An animal feeding study using genetically modified 'purple tomatoes' that express high levels of ACN and equivalent ACN-free red tomatoes was carried out which allowed direct assessment of the ability of dietary ACN to affect CVD risk. We used increasing concentrations of purple tomatoes $(2 \cdot 5,5$ and $10 \%)$ in chows containing a total of $10 \% \mathrm{w} / \mathrm{w} /$ tomato and compared the effects with a red tomato-only supplemented chow and a no tomato chow. This novel, highly controlled dietary intervention in the ApoE ${ }^{-1-} \mathrm{mouse}^{-}$ model assessed the dose-response effects of ACNs from tomato on atherosclerosis, inflammation and gene expression.

The $10 \%$ purple tomato diet caused a $45 \%$ mean reduction in aortic lesion size compared to the $10 \%$ red tomato control diet $(p<$ $0.001)$. Further, there was a very clear linear dose-response $\left(\mathrm{R}^{2}=0.999, p<0.0001\right.$ using average data $)$ showing that the dietary ACNs had beneficial effects by reducing atherosclerosis in this mouse model. Analysis of global gene expression data obtained from the livers and aorta of mice fed the $0 \%, 10 \%$ red and $10 \%$ purple tomato diets is undergoing and initial data is intended to be presented at the meeting. In conclusion, it is shown for the first time that consumption of tomato anthocyanins significantly reduces atherosclerosis in the $\mathrm{ApoE}^{-/-}$mouse model.

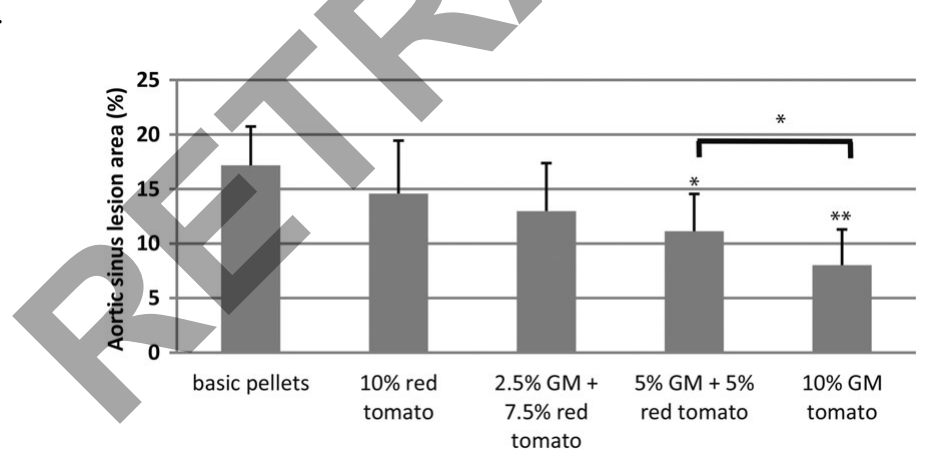

Fig. 1: All tomato-rich diets significantly reduced aortic sinus plaque area compared to the no tomato pellets group. Increasing concentrations of anthocyanin-rich tomatoes reduced plaque area significantly in a dose-dependent manner compared to red tomato $(p<0 \cdot 001)$. There were significant differences between the $10 \%$ red tomato and the $5 \%$ and $10 \%$ purple tomato groups $(p<0.05, p<0.001)$. Data was statistically analysed using one-way ANOVA coupled with Dunnett's multiple comparison test. Linear regression analysis was undertaken to test for a dose response. All values are given as means $\pm \mathrm{SD}(n=20)$. * $p<0.05 ; * * * p<0.001$ compared to $10 \%$ red tomato diet.

This research was funded by the European Commission (FP7 ATHENA, Grant No. 245121) and by the Biotechnology and Biological Sciences Research Council (UK; grant No. BB/J004545/1). 